\title{
Marine habitats - promising sources of novel microoganisms and new natural products with biotechnological applications
}

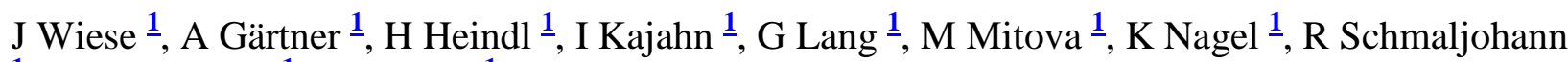
$\underline{1}$, I Schneemann $\underline{1}$, JF Imhoff $\underline{1}$

- ${ }^{1}$ Kieler Wirkstoffzentrum KiWiZ at IFM-GEOMAR, Am Kiel Kanal 44, 24106 Kiel, Germany

- Kongressbeitrag

Marine environments like surfaces of macroorganisms (i.e. algae, sponges, bryozoa, corals) or deep sea habitats provide excellent ecological niches for bacteria and fungi producing new natural products. Therefore, major goals of our projects are the isolation, identification and fermentation of marine-derived bacteria and fungi as well as extraction, structure elucidation and the determination of the biological activity of their metabolites. In addition, studies concerning the interactions between marine microorganisms and the microbial interactions with their hosts are in our focus.

We will present recent results from our work. Two recently described new species Tenacibaculum adriaticum isolated from a bryozoan and Amphritea atlantica (representing a new genus) derived from a deep sea mussel, both display antibiotic activities. Sponge-associated bacteria like Bacillus subtilis or Streptomyces sp. were identified as producers of new bacillaenes and phenazines, respectively. Interestingly, subinhibitory concentrations of microbial metabolites induced the production of new phenazines, which inhibited the growth of bacteria and different tumoral cell lines. The examination of fungal isolates revealed Penicillium sp. as the producer of new pentaens. The biosynthesis of these substances was studied by feeding experiments with ${ }^{13} \mathrm{C}$ labelled substances. Genetic approaches were used to select bacterial strains showing genes encoding for polyketide-synthase (PKS) and non-ribosomal peptide-synthetase (NRPS). These strains were selected for analysis of biological active new metabolites.

In conclusion, the exploitation of our unique strain collections offers great chances to find new natural products with possible application in human health, crop protection and other applied fields. 